Supporting Information for Publication

\title{
Photopatterning of Hydrogel Microarrays in Closed
}

\section{Microchips}

Burcu Gumuscu*, Johan G. Bomer, Albert van den Berg and Jan C.T. Eijkel**

BIOS Lab-on-a-Chip Group, MESA + Institute for Nanotechnology, MIRA Institute for Biomedical Technology and Technical Medicine, University of Twente, Enschede, The Netherlands 


\section{Supporting Information Captions}

S1. Phase contrast microscopy image of photopolymerized polyacrylamide hydrogel. Gray squares are glass pillars and blue squares are hydrogels. (a) Photopolymerization using an unpolished upper glass layer. (b) Photopolymerization using a polished upper glass layer. (c) Photomask patterns used in photopolymerization.

S2. (a) Phase contrast microscopy image of photopolymerized polyacrylamide hydrogel. Gray squares are glass pillars and blue squares are hydrogel. Dashed line presents the location where SEM image was obtained. Scale bar is $10 \mu \mathrm{m}$. (b) A scanning electron micrograph of a polyacrylamide hydrogel block that is photopolymerized between two glass pillars and firmly attached to the silanized glass layers.

S3. (a) Photopolymerization reaction of polyacrylamide. (b) Photopolymerization reaction of PEG DA.

S4. Schematic diagram of the crosslinking reaction in the hydrogel under (a) high and (b) low intensity UV illumination.

S5. The temperature profile of the microchip during intermittent and continuous illumination. The temperature profiles of polyacrylamide and PEG DA are very close to each other, while the amount of temperature rise is the same. The polymerization results are shown for PEG DA.

S6. Photoinitiator concentration versus the patterning resolution. Patterning resolution is described by NP (no polymerization) and $\pm \%$ (similarity percentage of the hydrogel patterns compared to the structures on the photomask), based on observations under the microscope. All experiments were duplicated, $n=2$. 


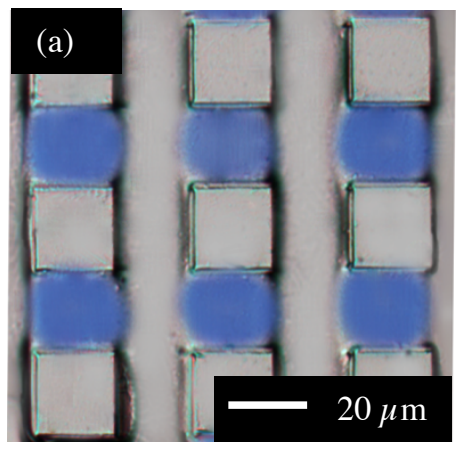

Hydrogel
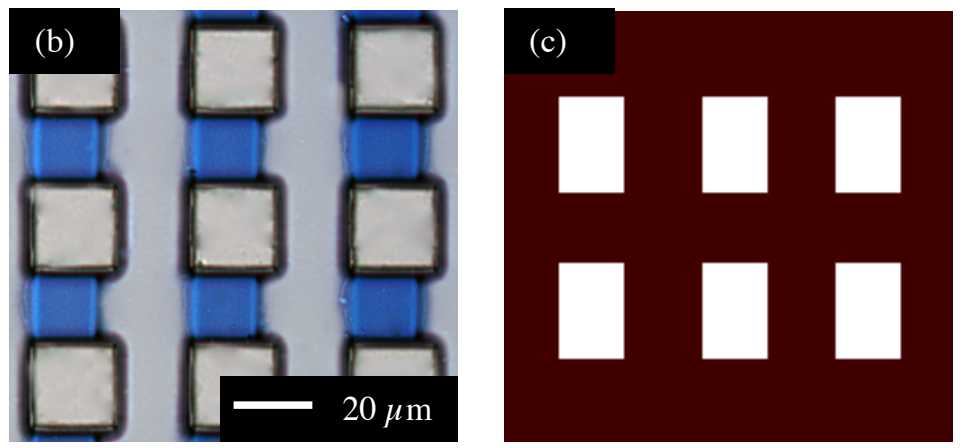

Air $\square$ Glass pillar

S1. Phase contrast microscopy image of photopolymerized polyacrylamide hydrogel. Gray squares are glass pillars and blue squares are hydrogels. (a) Photopolymerization using an unpolished upper glass layer. (b) Photopolymerization using a polished upper glass layer. (c) Photomask patterns used in photopolymerization. 


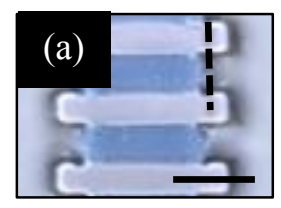

\section{(b) \\ upper glass layer}

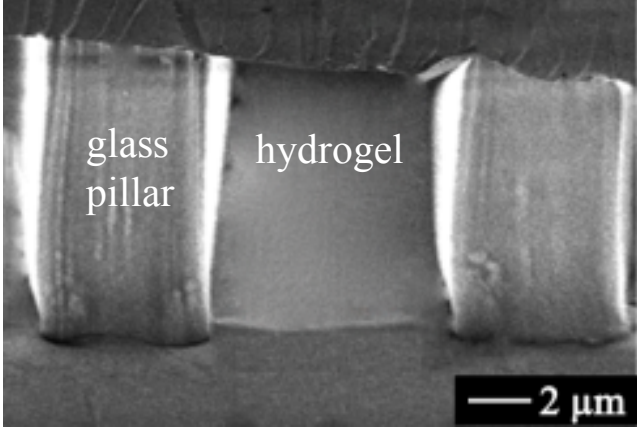

S2. (a) Phase contrast microscopy image of photopolymerized polyacrylamide hydrogel. Light gray rectangles are glass pillars and blue rectangles are hydrogel. Dashed line presents the location where SEM image was obtained. Scale bar is $10 \mu \mathrm{m}$. (b) A scanning electron micrograph of a polyacrylamide hydrogel block that is photopolymerized between two glass pillars and firmly attached to the silanized glass layers. 


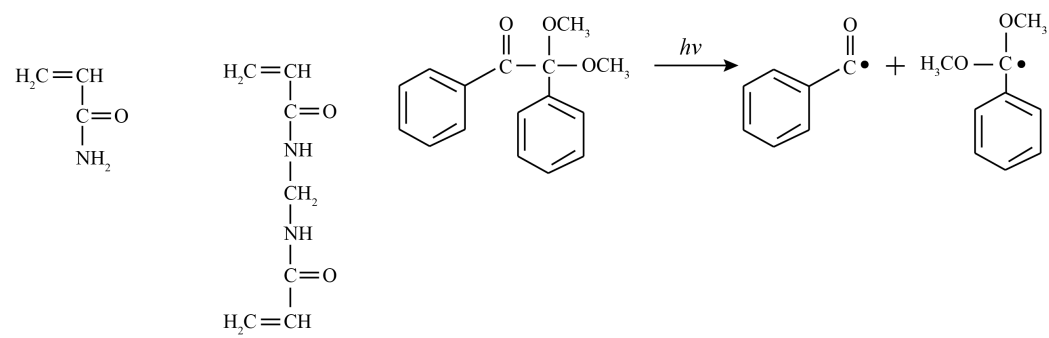

Acrylamide

Bis

DMPA

Activated DMPA

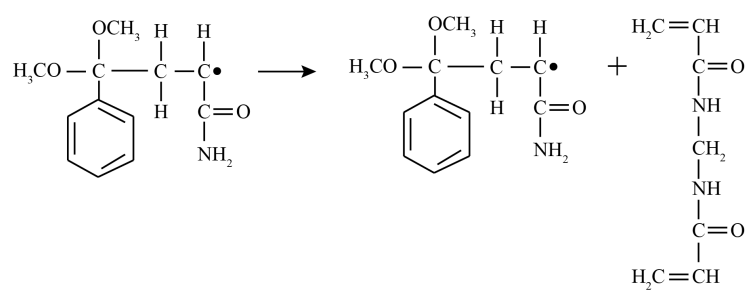

Activation

Initiation

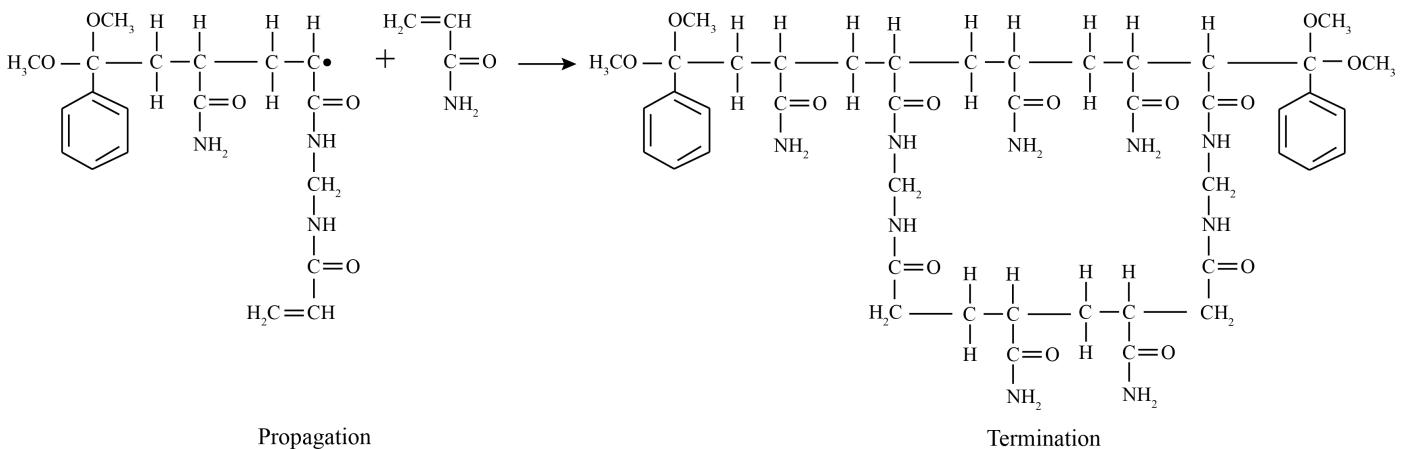

S3. (a) Photopolymerization reaction of polyacrylamide. 


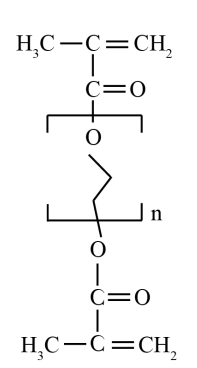

PEG Monomer<smiles>CC#CCCO</smiles>

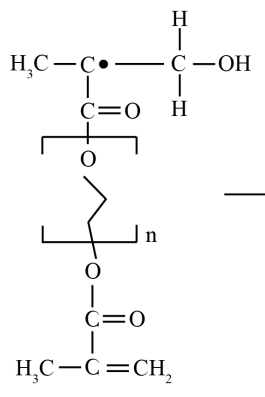

Activation

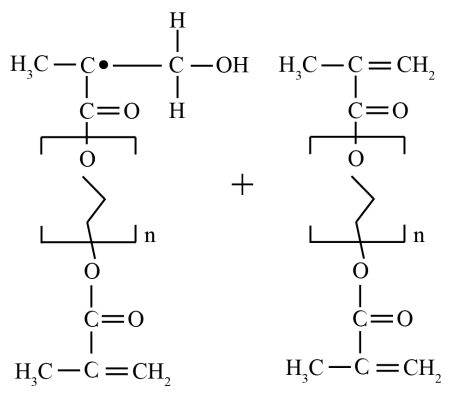

Initiation

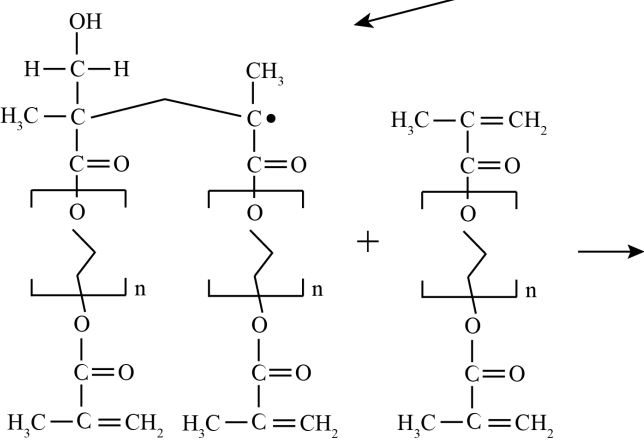

Propagation

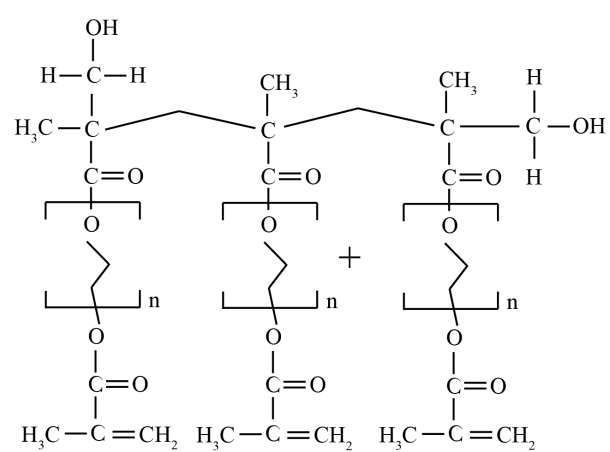

Termination

S3. (b) Photopolymerization reaction of PEG DA. 

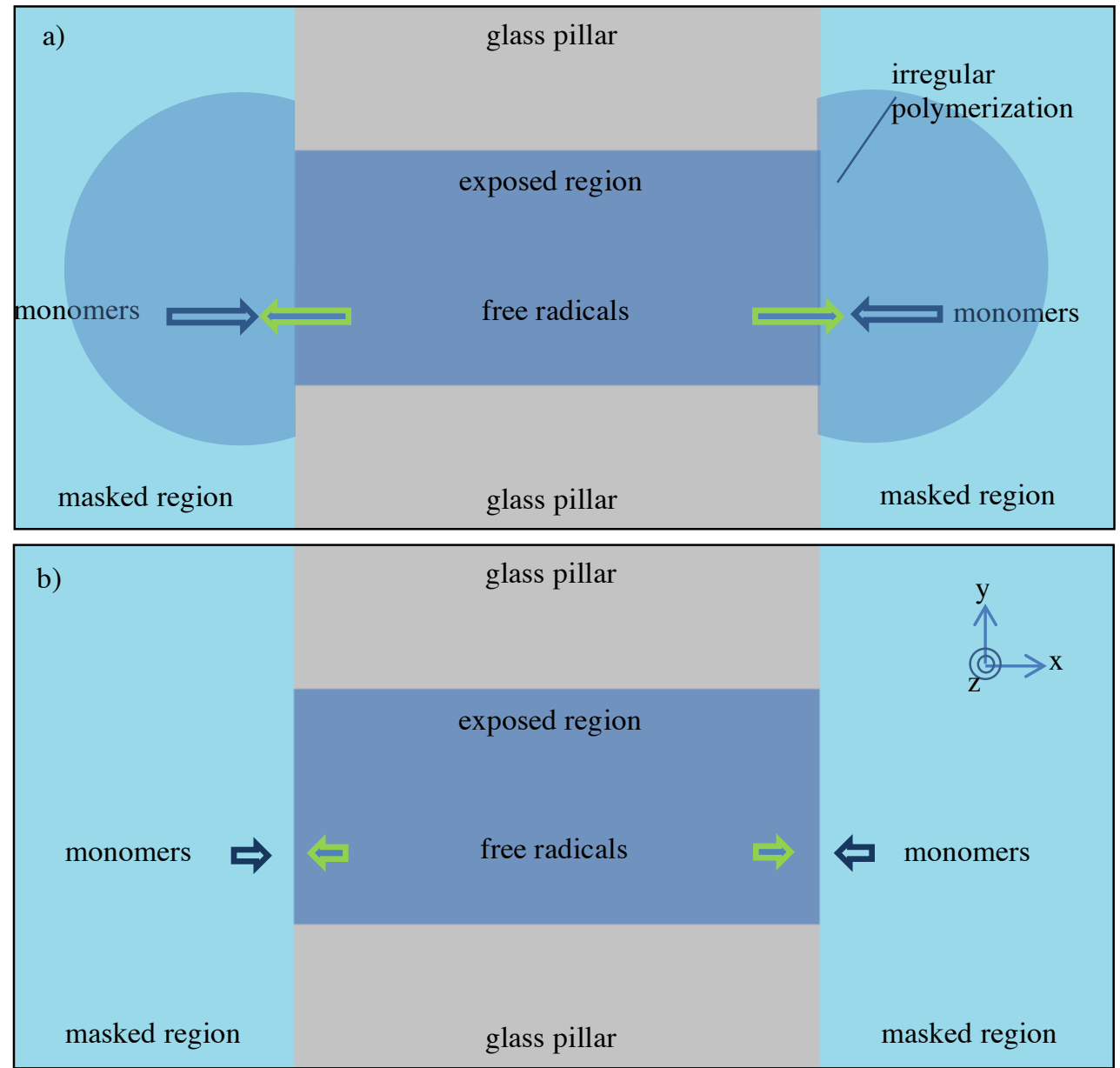

S4. Schematic diagram of the crosslinking reaction in the hydrogel under (a) high and (b) low intensity UV illumination. 

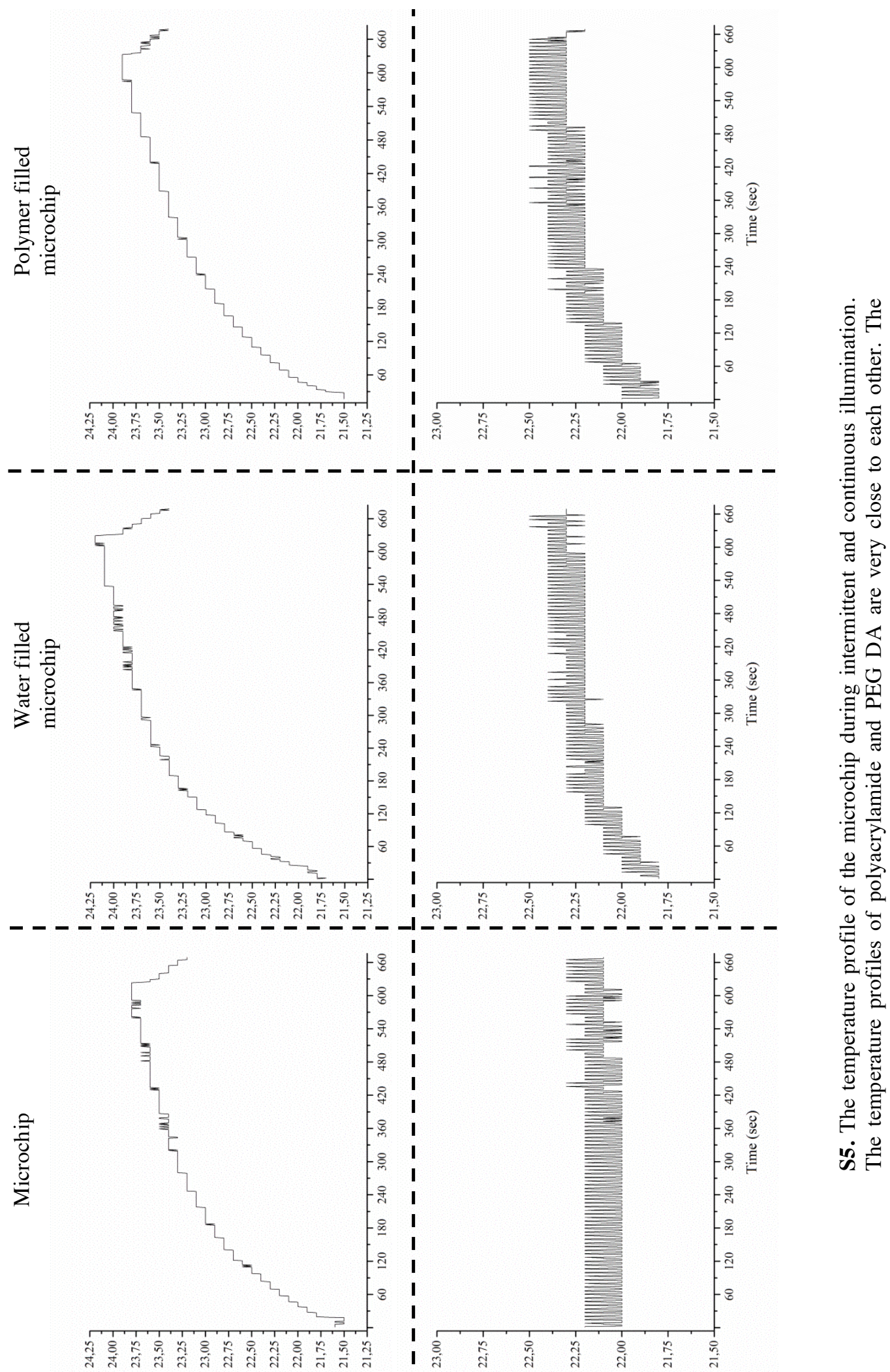

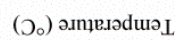

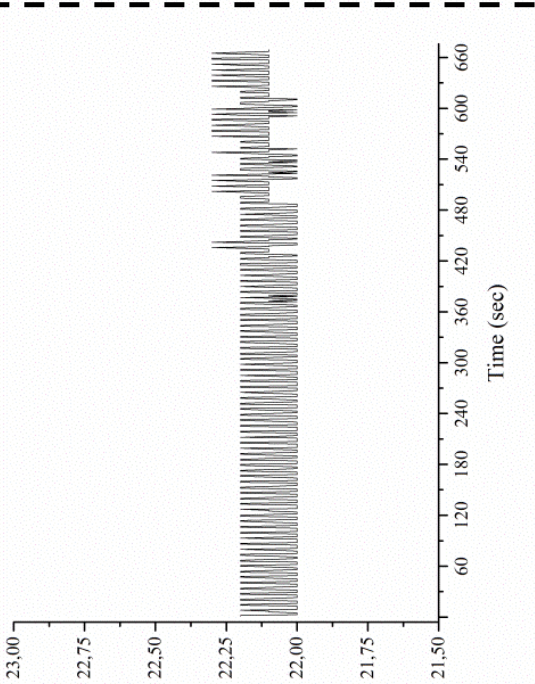

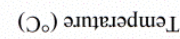

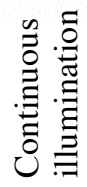

离.气气 
S6. Photoinitiator concentration versus the patterning resolution. Patterning resolution is described by NP (no polymerization) and $\pm \%$ (similarity percentage of the hydrogel patterns compared to the structures on the photomask), based on observations under the microscope. All experiments were duplicated, $\mathrm{n}=2$.

\section{a) Polyacrylamide}

\begin{tabular}{|c|c|c|c|c|c|}
\hline $\begin{array}{c}\text { monomer } \\
\text { concentration } \\
(\mathrm{T} \%)\end{array}$ & dmpa (\%) & $\begin{array}{c}\text { patterning } \\
\text { similarity to the } \\
\text { photomask }\end{array}$ & $\begin{array}{c}\text { monomer } \\
\text { concentration } \\
(\%)\end{array}$ & $\begin{array}{c}\text { irgacure } \\
(\%)\end{array}$ & $\begin{array}{c}\text { patterning } \\
\text { similarity to } \\
\text { the photomask }\end{array}$ \\
\hline \multirow[t]{4}{*}{2.5} & 1 & NP & 5 & 1 & NP \\
\hline & 2 & $\pm 70 \%$ & & 5 & NP \\
\hline & 5 & $\pm 75 \%$ & & 10 & $\pm 75 \%$ \\
\hline & 10 & $\pm 80 \%$ & & 15 & $\pm 80 \%$ \\
\hline \multirow[t]{4}{*}{4} & 1 & NP & 10 & 1 & NP \\
\hline & 2 & $\pm 70 \%$ & & 5 & NP \\
\hline & 5 & $\pm 80 \%$ & & 10 & $\pm 97 \%$ \\
\hline & 10 & $\pm 80 \%$ & & 15 & $\pm 97 \%$ \\
\hline \multirow[t]{4}{*}{6} & 1 & NP & 15 & 1 & NP \\
\hline & 2 & $\pm 65 \%$ & & 5 & NP \\
\hline & 5 & $\pm 80 \%$ & & 10 & $\pm 95 \%$ \\
\hline & 10 & $\pm 95 \%$ & & 15 & $\pm 97 \%$ \\
\hline \multirow[t]{4}{*}{8} & 1 & NP & 20 & 1 & NP \\
\hline & 2 & $\pm 70 \%$ & & 5 & NP \\
\hline & 5 & $\pm 90 \%$ & & 10 & $\pm 97 \%$ \\
\hline & 10 & $\pm 99 \%$ & & 15 & $\pm 99 \%$ \\
\hline
\end{tabular}

